\title{
CCD Photometry and Spectroscopy of 14 IRAS Sources having Far-IR Colours Similar to Planetary Nebulae
}

\author{
B.E. Reddy and M. Parthasarathy
}

Indian Institute of Astrophysics

CCD imaging and BVRI photometry of 14 IRAS sources with far-IR colours similar to planetary nebulae and post-AGB stars are presented. Also results of optical and near-IR spectroscopy of 10 of these candidates are given. Based on the spectral energy distribution from $0.4 \mu \mathrm{m}$ to $100 \mu \mathrm{m}$, the sample of program stars are put into two groups. The sources IRAS 08187-1905, IRAS 05238-0626 and IRAS 17086-2403 present similar flux distributions. These three sources have detached cold dust components with dust radii $\mathrm{R}_{d} \approx$ $1000 R_{\star}$. The low infrared variability of theses sources suggests that the intense mass loss has been ceased. All three sources are at high galactic latitude $\left(1>9^{\circ}\right)$ suggesting that these are old low-mass evolved stars. In the IRAS colour-colour diagram of Likkel et al (1991) these sources fall in the region where most of the stars are evolved stars and PNe but without CO detection. This is consistent with at least one source IRAS 17086-2403, in which $\mathrm{OH}$ and $\mathrm{CO}$ molecular features are not detected. The far-IR excess, non-variability and high latitude of these objects suggest that these are post-AGB supergiants, slowly evolving towards planetary nebula phase.

The rest of the sources in the sample: IRAS $04296+3429$, IRAS $05113+1347$, IRAS 05341+0852, IRAS 06530-0213, IRAS 07253-2001, IRAS 08143-4406, IRAS 14429-4539, IRAS 17150-3224, IRAS 17291-2402, IRAS 17441-2411 and IRAS 23304+6147 present a double peak energy distribution. One peak represents the stellar emission, obscured by optically thin hot dust component and the other is produced by the reemission in the farIR of the stellar radiation obscured by the dust envelope. The presence of hot and cold dust components suggest that there was a discontinuity in the mass loss history. A possible cause for such discontinuity may be periodic thermal pulsations predicted for the AGB model stars ( Iben and Renzini 1983). Most of the sources in this second group are classified as carbon-rich based on the infrared properties of their dust envelopes. The carbonrich dust envelopes imply the overabundance of carbon in the photospheres, which suggest that these stars have undergone third dredge-up which occurs in the advanced phase of AGB evolution of low and intermediate mass stars.

The analysis of low resolution optical spectra, BVRI photometry and the comparision of observed flux distribution with kurucz models show that most of these sources are $F$ and $G$ post-AGB supergiants.

\section{REFERENCES}

Iben I., Jr., Renzini A. 1983, Ann. Rev. Astron. Astrophys. 21, 271

Likkel L., Forveille T., Omont A. and Morris M. 1991, A\&A 246, 153

Omont A., Loup C., Forveille T., te Lintel Hekkert P., Habing H. and Sivagnanam P. 1993, A\&A 267, 515 